\title{
The effect of compressive loading magnitude on in situ chondrocyte calcium signaling
}

\author{
Ryan M. J. Madden · Sang-Kuy Han · Walter Herzog
}

Received: 11 December 2013 / Accepted: 6 May 2014 / Published online: 23 May 2014

(c) The Author(s) 2014. This article is published with open access at Springerlink.com

\begin{abstract}
Chondrocyte metabolism is stimulated by deformation and is associated with structural changes in the cartilage extracellular matrix (ECM), suggesting that these cells are involved in maintaining tissue health and integrity. Calcium signaling is an initial step in chondrocyte mechanotransduction that has been linked to many cellular processes. Previous studies using isolated chondrocytes proposed loading magnitude as an important factor regulating this response. However, calcium signaling in the intact cartilage differs compared to isolated cells. The purpose of this study was to investigate the effect of loading magnitude on chondrocyte calcium signaling in intact cartilage. We hypothesized that the percentage of cells exhibiting at least one calcium signal increases with increasing load. Fully intact rabbit femoral condyle and patellar bone/cartilage samples were incubated in calcium-sensitive dyes and imaged continuously under compressive loads of $10-40 \%$ strain. Calcium signaling was primarily associated with the dynamic loading phase and greatly increased beyond a threshold deformation of about $10 \%$ nominal tissue strain. There was a trend toward more cells exhibiting calcium signaling as loading magnitude increased ( $p=0.133$ ). These results provide novel information toward identifying mechanisms underlying calciumdependent signaling pathways related to cartilage homeostasis and possibly the onset and progression of osteoarthritis.
\end{abstract}

R. M. J. Madden $(\bowtie) \cdot$ W. Herzog

Human Performance Laboratory, University of Calgary, 2500

University Dr. NW, Calgary, ABT2N 1N4, Canada

e-mail: r.madden@ucalgary.ca

W. Herzog

e-mail: wherzog@ucalgary.ca

S.-K. Han

Advanced Biomedical \& Smart Welfare Technology R\&BD

Group, Korea Institute of Industrial Technology,

Cheonan-si 331-822, Chungcheongnam-do, Republic of Korea
Keywords Articular cartilage $\cdot$ Chondrocyte $\cdot$ Calcium signaling $\cdot$ Mechanobiology $\cdot$ Mechanotransduction

\section{Introduction}

Osteoarthritis (OA) is a degenerative joint disease characterized by a breakdown of the articular cartilage extracellular matrix $(\mathrm{ECM})$ resulting in joint pain, inflammation, and stiffness. Currently, the mechanisms underlying the onset and progression of OA are not well understood. Under normal physiological conditions, joint loading causes deformation of the cartilage and its cells (Abusara et al. 2011). This deformation causes a complex array of biological processes to occur resulting in the synthesis and/or degradation of structural macromolecules (Sah et al. 1989). These processes have been associated with the adaptive/degenerative changes involved in OA (Wieland et al. 2005). Despite this knowledge, the complex processes behind cell mechanotransduction remain to be elucidated. One of the initial steps is an influx of calcium into the cell cytoplasm known as calcium signaling (Guilak et al. 1999). Calcium is a second messenger directly involved in many cellular processes including gene transcription, contraction, and proliferation (Bootman et al. 2001). It can enter the cytoplasm from extracellular stores through the activation of various membrane channels or from intracellular stores, such as the endoplasmic reticulum, which releases calcium via channels such as the inositol-1,4,5-triphosphate (IP3R) receptor (Berridge 1993; Berridge et al. 2003; Huser and Davies 2007).

For chondrocytes, calcium is involved in matrix synthesis (Clark et al. 1994; Valhmu and Raia 2002), cytoskeletal remodeling (Erickson et al. 2003; Millward-Sadler and Salter 2004), cell hyperpolarization (Wright et al. 1996), and cell death (Huser and Davies 2007; Amin et al. 2009). 
Previous work investigating chondrocyte calcium signaling has focused on cells removed from their physiological environment, such as isolated cells/cell cultures (Yellowley et al. 1997; Guilak et al. 1999; Mizuno 2005; Chao et al. 2006; Kono et al. 2006), and chondrocytes embedded in gel constructs (Roberts et al. 2001; Pingguan-Murphy et al. 2005, 2006). It was recently shown that calcium signaling of chondrocytes in situ differs compared to isolated cells embedded in gel constructs. Specifically, calcium signaling in situ occurred virtually instantaneously with loading as compared to the time-delayed signaling previously observed in cell-gel constructs (Han et al. 2012). The duration of calcium signals observed in in situ chondrocytes was also shorter than signals observed in cell-gel constructs and completely isolated cells and instead were closer to the durations of osmotically induced calcium signals in intact mouse femora (Han et al. 2012). These results suggest that the calcium signaling behavior of chondrocytes in the intact tissue differs substantially from isolated cells and cell-gel constructs. Therefore, further investigation is needed to understand chondrocyte mechanotransduction as it occurs in intact cartilage.

Loading magnitude is thought to play a key role in the calcium signaling response of chondrocytes (Yellowley et al. 1997; Guilak et al. 1999), and the magnitude of mechanical stimuli has been shown to greatly influence $\mathrm{Ca}^{2+}$ signaling in other cell types, such as endothelial cells (Shen et al. 1992) and osteocytes (Lu et al. 2012). We have shown previously that local ECM strains and chondrocyte deformations induced by mechanical loading differ between joint regions (Madden et al. 2013) and others have observed topographical variations in aggrecan synthesis (Little and Ghosh 1997), a process that involves calcium signaling (Fitzgerald et al. 2004). Therefore, the purpose of this study was to examine the effect of compressive loading magnitude on chondrocyte calcium signaling in intact cartilage attached to its native bone for two distinct joint regions. A secondary objective was to relate previously measured local ECM strains and chondrocyte deformations (Madden et al. 2013) to the observed calcium response. We hypothesized that the percentage of cells exhibiting at least one calcium response would increase with increasing compressive tissue load.

\section{Methods}

\subsection{Sample preparation}

Cartilage samples were obtained from the knees of six to eight-month-old New Zealand White rabbits $(n=5)$. All experiments were approved by the Animal Ethics Committee of the University of Calgary. Femoral condyles $(n=10)$ and patellae $(n=5)$ were extracted from the knee joints, stripped of non-cartilaginous connective tissues while main- taining the underlying bone, and placed in high glucose Dulbecco's Modified Eagle's Medium with $4 \mathrm{mM}$ L-glutamine and $25 \mathrm{mM}$ HEPES (DMEM, Gibco, OR, USA) supplemented with $1 \%$ penicillin/streptomycin and $1 \mathrm{mM}$ sodium pyruvate. Tissue thickness of each sample was measured by needle indentation at two locations close to the region to be loaded. Cartilage samples were then incubated in medium containing the ratiometric calcium-sensitive dyes Fura Red $(30 \mu \mathrm{M})$ and Fluo-4 $(15 \mu \mathrm{M})$ for $1 \mathrm{~h}$ at $37^{\circ} \mathrm{C}$. After staining, the samples were rinsed in dye-free medium for $20 \mathrm{~min}$ prior to testing. All specimens were tested within $15 \mathrm{~h}$ of collection, and the order of testing was randomized to mitigate the effect of time.

\subsection{Mechanical testing and confocal imaging}

Samples were embedded in dental cement and placed into the chamber of a custom-designed in situ indentation system (Fig. 1) mounted to the stage of a confocal laser scanning microscope (LSM 510, Zeiss Inc., Germany). Briefly, the system consists of a light-transmissible indenter, piezoactuator, load cell, and displacement transducer (Han et al. 2009). The specimen height was adjusted to the point of initial contact between the indenter and cartilage surface, and samples were positioned as described previously (Han et al. 2010, 2012; Madden et al. 2013). A series of static loads were applied to the samples in the following order: 10, 20, 30 and $40 \%$ compressive strain (Fig. 2). Loading was applied using a light-transmissible cylindrical indenter $($ diameter $=2 \mathrm{~mm})$ at an average rate of $0.4 \pm 0.2 \% / \mathrm{s}(1.8 \pm 1.1 \mu \mathrm{m} / \mathrm{s})$ and controlled by a custom-written program (LabVIEW, National Instruments, USA). After the loading ramp was complete, displacements were held for three minutes as previous work has shown that the majority of in situ chondrocyte calcium signaling occurs during and immediately following the loading ramp (Han et al. 2012).

Prior to loading, a characteristic group of cells was identified in the field of view and its position marked on the
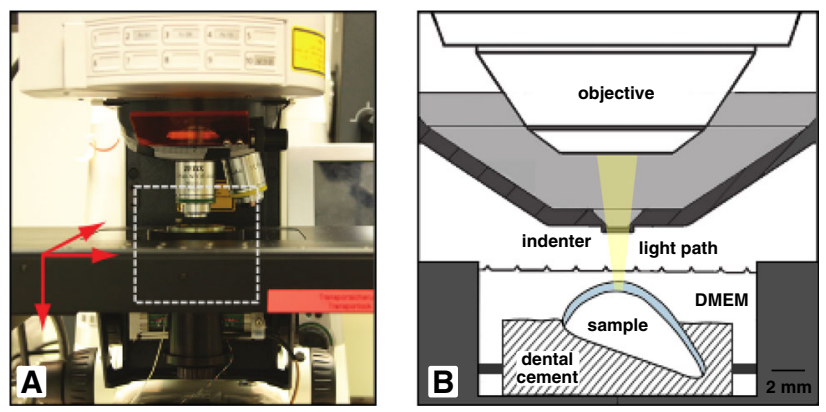

Fig. 1 a Assembled view of the system for simultaneous loading and imaging of in situ chondrocytes. $\mathbf{b}$ Schematic of dashed white box shown in a showing the orientation of the sample in the tissue holder, the indenter, and the light path of the confocal microscope 


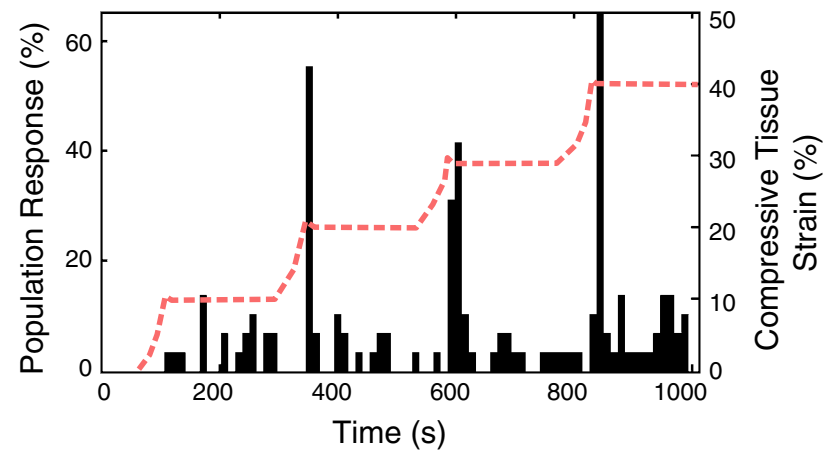

Fig. 2 Black bars percentage of cells exhibiting a calcium signal for a representative sample $(1 \mathrm{bar}=3$ image frames $=12 \mathrm{~s})$. The dashed red line shows the applied loading protocol on the secondary $y$ axis

screen. This was done to ensure that the same cells were imaged throughout the entire loading protocol. Confocal images were captured through a 40x 0.8 NA $0.17 \mathrm{~mm}$ cover glass-corrected water immersion objective (Zeiss Inc., Germany). The image volume collected was $297 \times 297 \times 10 \mu \mathrm{m}^{3}$ at a resolution of $0.58 \mu \mathrm{m} /$ pixel in the $x-y$ plane (Fig. 3a). Confocal time series images were collected continuously at $0.25 \mathrm{~Hz}$ starting $60 \mathrm{~s}$ before application of the first load (eg. $t=0 \mathrm{~s}$ in Fig. 2) until completion of the holding period of the final load (eg. $t=1000 \mathrm{~s}$ in Fig. 2). The number of superficial zone chondrocytes imaged was $82 \pm 6$ (min: 37 , max: 108) and $92 \pm 7$ (min: 69, max: 106) for femoral condyles and patellae samples, respectively. Specimen temperature was maintained at $37^{\circ} \mathrm{C}$ for the duration of the experiments.

\subsection{Confocal image analysis}

The ratio of fluorescence intensity between Fura Red and Fluo-4 was normalized to the central moving average for each chondrocyte using a $60 \mathrm{~s}$ window. Calcium signals were defined as an increase in baseline levels greater than 10 times the standard deviation of the normalized ratio (Fig. 3b) (Han et al. 2012). The duration and peak amplitude of each calcium signal was determined. Response time was defined as
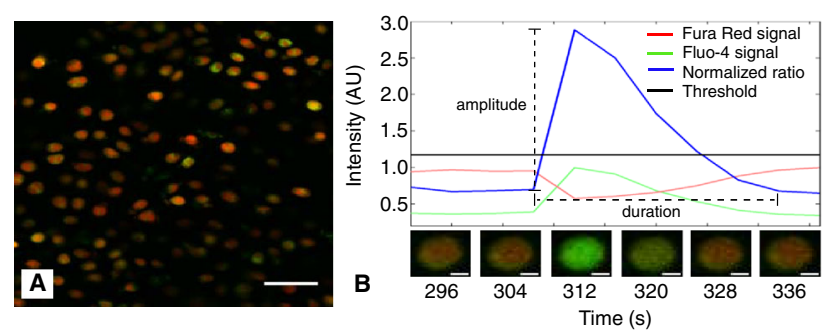

Fig. 3 a Exemplar field of view ( $x-y$ plane) showing fluorescently labeled cells (scale bar $=50 \mu \mathrm{m}$ ). b Calcium signal of a representative cell indicating the cell-specific threshold, signal characteristics, and corresponding confocal images (scale bars $=5 \mu \mathrm{m}$ ) the time from the onset of the loading ramp to the frame in which the most calcium signaling peaks were observed. This was done at each load and for each sample to compare the time response of calcium signaling as a function of loading magnitude. The population response of a sample was defined as the percentage of cells exhibiting at least one calcium signal over a specified period. The fraction of signals occurring at each load was expressed as a percentage of the total number of signals that occurred over the duration the experiment.

\subsection{Statistical analysis}

Population response, signal fraction, and time response ( $n=10$ femoral condyles and $n=5$ patellae) were examined using two-way repeated-measures ANOVA (SPSS 20, SPSS Inc., IL, USA) with joint region and load as the betweenand within-subject factors, respectively $(\alpha=0.05)$. Calcium signal amplitude and duration $(n=946$ signals from the 10 femoral condyles and $n=751$ signals from the 5 patellae) were analyzed using generalized estimating equations to determine the effect of joint region, loading magnitude, and interaction effects $(\alpha=0.05)$ while accounting for multiple responses from the same cells and to account for the unbalanced design. Bonferroni post-hoc adjustments were conducted when appropriate. Results are presented as means \pm 1 standard error of the mean.

\section{Results}

\subsection{Population response}

Figure 4 shows the population response of in situ chondrocytes over the entire experiment. During the initial $60 \mathrm{~s}$ unloaded imaging period, little to no calcium signaling

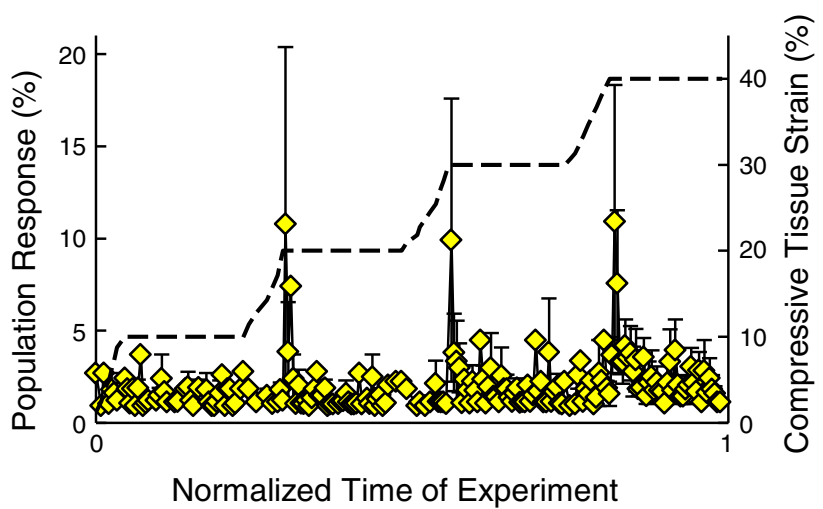

Fig. 4 Average calcium signaling response of all femoral condyle samples over the duration of the experiments. The dashed black line shows the applied load on the secondary $y$ axis. Results are presented as means \pm 1 standard error of the mean 


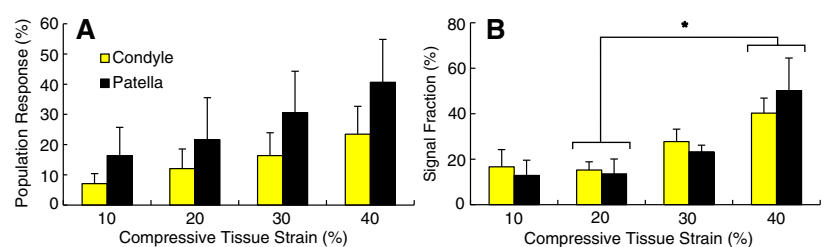

Fig. 5 a Average calcium signaling response of all femoral condyle and patellar samples during each loading phase. b Fraction of signals occurring at each load level relative to the total number of signals occurring for each joint region (946 signals from 10 femoral condyles, 751 signals from 5 patellae). Results are presented as means \pm 1 standard error of the mean. Star indicates a significant difference in pooled samples $(p<0.05)$

activity was observed for all samples. Compressive loading of cartilage resulted in a calcium signal response in $32 \pm 9 \%$ of femoral condyle cells and $58 \pm 16 \%$ of patellar cells. The majority of calcium signals occurred during or immediately following the transient phase of loading (Fig. 4). The average response time to peak calcium signaling was $77 \pm 8 \mathrm{~s}$ and $74 \pm 10$ s for femoral condyles and patellae, respectively, and generally decreased with increasing compressive tissue strain (Table 1). Figure 5a shows the population response of the chondrocytes at each load, defined as any cell that signaled between the start of the loading ramp for the load of interest and before the start of the next loading ramp. The percentage of chondrocytes responding with at least one calcium signaling event tended to increase with increasing load for both joint regions (Fig. 5a; Table 1). Patellar samples exhibited a trend toward greater population response at each load compared to the femoral condyles, although these differences did not reach statistical significance ( $p>0.05 ;$ Fig. 5a). For a given load, more responding chondrocytes exhibited a single calcium signaling event compared to multiple signals, except for the patellar samples at $20 \%$ tissue strain.

While the main effect of loading magnitude did not reach statistical significance, there was a trend toward an increase in the population response with increasing load ( $p=0.133$, Fig. 5a). Additionally, more calcium signaling events occurred at $40 \%$ tissue strain; this difference was significant when compared to the $20 \%$ tissue strain condition $(p<0.05$, Fig. $5 b)$ and was close to significance compared to the 10 and $30 \%$ tissue strain conditions $(p=0.142$ and 0.120 , respectively).

\subsection{Calcium signal characteristics}

Both loading magnitude and joint region had a significant effect on calcium signal amplitude $(p<0.05$ and $p<0.001$, respectively). The calcium signal amplitude of femoral condyle chondrocytes remained consistent for the $10-30 \%$ tissue strain conditions, but then decreased signifi-
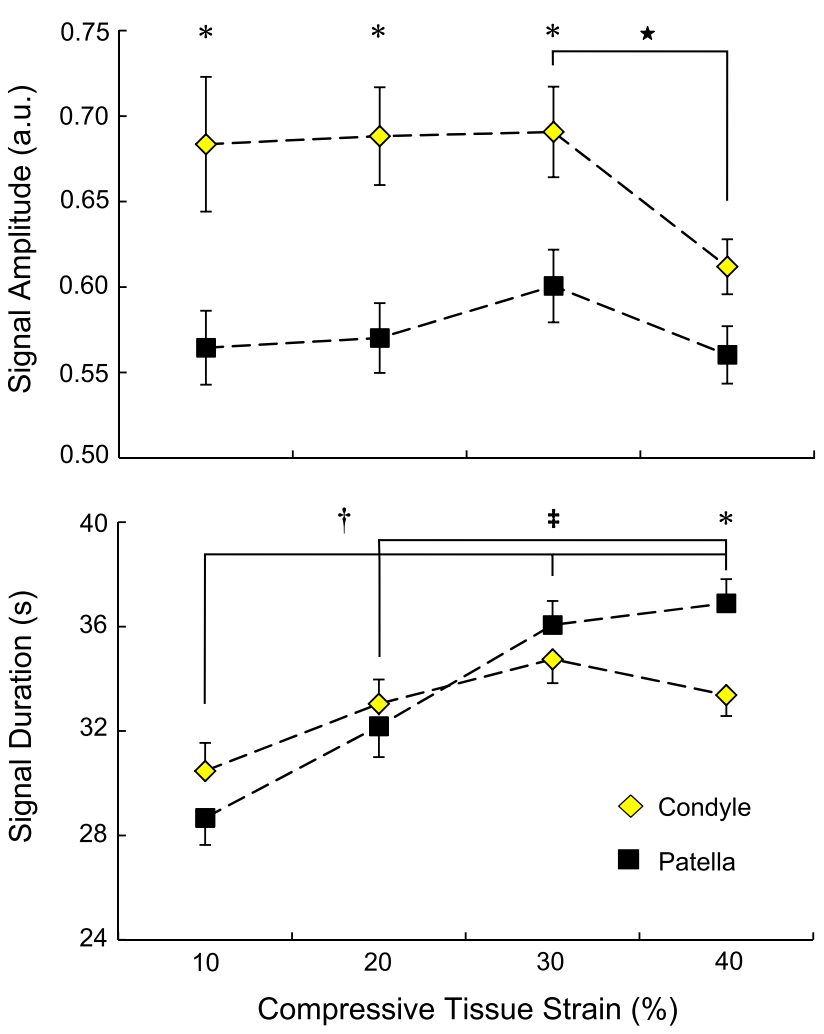

Fig. 6 Calcium signal characteristics for chondrocytes from femoral condyles (yellow diamonds, $n=946$ signals from 10 samples) and patellae (black squares, $n=751$ signals from 5 samples). Results are presented as means \pm 1 standard error of the mean. Asterisk indicates a significant difference between femoral condyles and patellae at a given load $(p<0.05)$. Star indicates a significant difference within femoral condyles between the indicated loads $(p<0.05)$. $\dagger$ and $\$$ indicate significant differences within patellae between the indicated loads $(p<$ 0.001 and 0.05 , respectively)

cantly at $40 \%$ strain ( $p<0.05$, Fig. 6). No significant change in signal amplitude was observed for patellar chondrocytes across all tissue strain conditions (10-40\%), although there was a slight decrease at $40 \%$ strain, similar to the finding made for the femoral condyle cells. The signal amplitude of patellar cells was significantly less than femoral condyle cells for the 10-30\% tissue strain conditions $(p<0.05$, Fig. 6) and close to significance at $40 \%(p=0.052)$.

Loading magnitude had a significant effect on the duration of calcium signals $(p<0.001)$. For femoral condyle cells, signal duration remained relatively constant across all tissue loads, with a near-significant increase in signal duration at $30 \%$ compared to $10 \%$ tissue strain $(p=0.050)$. In contrast, patellar chondrocytes exhibited steadily increasing signal durations as the tissue strain was increased (Fig. 6; Table 1). At $40 \%$ tissue strain, the average calcium signal duration of patellar cells was significantly greater than that measured for femoral condyle cells ( $p<0.05$, Fig. 6). 
Table 1 Summary of calcium signaling event data obtained from mechanical compression tests of in situ chondrocytes

\begin{tabular}{|c|c|c|c|c|c|}
\hline & \multirow{2}{*}{$\begin{array}{l}\text { Joint } \\
\text { Region }\end{array}$} & \multicolumn{4}{|c|}{ Applied tissue strain (\%, compressive) } \\
\hline & & 10 & 20 & 30 & 40 \\
\hline Population response $^{\mathrm{a}}$ & COND & $7.1 \pm 3.3$ & $12.1 \pm 6.5$ & $16.4 \pm 7.6$ & $23.5 \pm 9.2$ \\
\hline$(\%)$ & PAT & $16.4 \pm 9.4$ & $21.6 \pm 13.9$ & $30.6 \pm 13.7$ & $40.6 \pm 14.2$ \\
\hline Signal fraction ${ }^{\mathrm{b}}$ & COND & $16.7 \pm 7.6$ & $15.3 \pm 3.6$ & $27.8 \pm 5.4$ & $40.3 \pm 6.6$ \\
\hline$(\%)$ & PAT & $12.9 \pm 6.7$ & $13.6 \pm 6.5$ & $23.3 \pm 2.9$ & $50.2 \pm 14.3$ \\
\hline Response time to peak ${ }^{\mathrm{a}}$ & COND & $93 \pm 22$ & $73 \pm 20$ & $78 \pm 12$ & $69 \pm 4$ \\
\hline (s) & PAT & $66 \pm 15$ & $80 \pm 26$ & $69 \pm 19$ & $80 \pm 21$ \\
\hline Local ECM strain ${ }^{c}$ & COND & $15 \pm 2$ & $23 \pm 2$ & $28 \pm 2$ & $34 \pm 2$ \\
\hline$(\% \text { compressive })^{*}$ & PAT & $16 \pm 4$ & $31 \pm 3$ & $39 \pm 4$ & $44 \pm 4$ \\
\hline Normalized cell volume $^{\mathrm{c}}$ & COND & $98 \pm 1$ & $96 \pm 1$ & $94 \pm 1$ & $92 \pm 1$ \\
\hline$(\%)^{*}$ & PAT & $102 \pm 2$ & $111 \pm 3$ & $111 \pm 3$ & $110 \pm 3$ \\
\hline
\end{tabular}

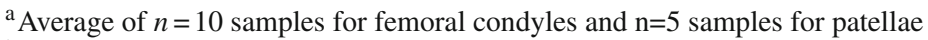

${ }^{\mathrm{b}}$ Average of $n=946$ signals from the 10 femoral condyles and $n=751$ signals from the 5 patellae

${ }^{\mathrm{c}}$ Measured in a previous study (Madden et al. 2013)

\section{Discussion}

The results of this study support previous observations that chondrocytes in the intact cartilage respond to mechanical compression by initiating intracellular calcium transients during and immediately following tissue compression (Han et al. 2012). A distinct trend was observed for an increase in calcium signaling activity with increasing compressive tissue strain ( $p=0.133$, Fig. 5a), thus confirming our hypothesis. Previous findings suggested that, following an initial mechanical stimulus, cells may become desensitized to further signaling, therefore the results observed here may underestimate the relationship between tissue loading and calcium signaling (Donahue et al. 2003; Han et al. 2012). This desensitization may be explained in part by the lack or defficiency of certain signaling pathways in chondrocytes, such as the T-type voltage-gated calcium channels which are associated with repetitive signaling in other cells types (Lu et al. 2012). Stretch-activated ion channels (SACs) likely play a key role in the mechanobiological response of chondrocytes (Guilak et al. 1999; Roberts et al. 2001; Mobasheri et al. 2002), and it is possible that the majority of these channels are recruited during the initial mechanical loading step. Receptor desensitization and ion channel fatigue are also factors that may contribute to the diminishing response with repeated mechanical loading (Ralevic and Burnstock 1998).

The time-course response of chondrocyte calcium signaling observed here $(76 \pm 9 \mathrm{~s}$ after onset of loading ramp) agrees well with previous in situ work $(\sim 35 \mathrm{~s}$; Han et al. 2012), and with observations of osmotically induced calcium signaling in intact mouse femora $(\sim 20 \mathrm{~s}$; Clark et al. 2010) and calcium signaling following direct application of hydrostatic fluid pressure to isolated superficial zone bovine chondrocytes ( $\sim 72 \mathrm{~s}$; Mizuno 2005). Conversely, the peak calcium response of chondrocytes embedded in gel constructs occurs much more slowly $(\sim 200 \mathrm{~s}$; Roberts et al. 2001). Therefore, while calcium signaling may be a downstream signaling event in cell-gel constructs, the results of the current study taken together with previous work on in situ chondrocytes (Clark et al. 2010; Han et al. 2012b) and isolated cells subjected to direct stimulation (Mizuno 2005) suggests that calcium signaling is an initial response in the chondrocyte mechanotransduction process in intact tissue. Since this response differs for cell-gel constructs, it is likely that interactions between the chondrocytes and their surrounding ECM plays a key role in this process. Furthermore, the majority of signaling observed here occurred during or immediately following the dynamic loading phase with minimal activity during the static hold period of each load (Fig. 4). Therefore, dynamic factors such as strain rate and fluid flow-induced shear stress may be more influential than loading magnitude in regulating the calcium response of chondrocytes to mechanical loading (Yellowley et al. 1997; Pingguan-Murphy et al. 2006). Primary cilia and transmembrane proteins such as integrins facilitate chondrocyte-ECM interaction, are highly responsive to fluid flow, and are directly involved in chondrocyte calcium signaling (Degala et al. 2011; Wann et al. 2012). Additionally, aggrecan expression following mechanical compression, which has been linked to calcium signaling (Fitzgerald et al. 2004), is highest in areas of high interstitial fluid flow (Buschmann et al. 1999). Future studies may utilize chemicals, such as integrin-specific Arg-Gly-Asp (RGD) peptides (Wright et al. 1997; Degala et al. 2011), to investigate the specific roles of these cellular components, determine the extent to which cell-ECM interactions regulate calcium sig- 
naling, and to elucidate which channels are involved in the associated signaling pathways for chondrocytes in intact cartilage.

At $10 \%$ applied tissue strain, $\mathrm{Ca}^{2+}$ signaling activity appeared generally scattered and disorganized, whereas at loads $\geq 20 \%$ tissue strain calcium signals occurred during or immediately after the transient loading phase with minimal activity during the static hold period (Fig. 4). This apparent synchronization of $\mathrm{Ca}^{2+}$ activity suggests that loads $\geq 20 \%$ nominal tissue strain may represent a mechanical threshold for chondrocytes with respect to cartilage homeostasis. It is interesting to note the significant increase in the fraction of calcium signals at $40 \%$ tissue strain (Fig. 5b), which corresponds to supra-physiological loading conditions (Herberhold et al. 1999). This finding could indicate that large compressive strains beyond physiological levels result in abnormal metabolic activity of the chondrocytes. Future studies could investigate this hypothesis by monitoring the regulation of genes associated with calcium signaling such as aggrecan, type II collagen, link protein, and matrix metalloproteinases (Fitzgerald et al. 2004) under the mechanical loads applied here to determine if the metabolic response is primarily anabolic or catabolic.

The population response of in situ chondrocytes under mechanical compression may also be related to the superficial zone ECM strain under mechanical compression. In a previous study under similar experimental conditions, the local ECM strain in the superficial zone of patellar cartilage was found to be greater than that in femoral condyles for a given nominal tissue strain (Table 1; Madden et al., 2013). In the current study, patellar samples exhibited a trend toward a greater percentage of cells responding with calcium signals for a given tissue load compared to femoral condyles (Fig. 5a), although these differences were not statistically significant likely due to the relatively small number of patellar samples. This observation provides further evidence implicating transmembrane proteins and receptors that interact with the ECM in the calcium response of chondrocytes in intact cartilage, as suggested by others (Guilak et al. 1999; Millward-Sadler et al. 1999; Loeser 2002; Mobasheri et al. 2002; Millward-Sadler and Salter 2004; Degala et al. 2011, 2012; Wann et al. 2012).

Furthermore, calcium signaling has been linked to changes in cell volume and membrane expansion (Guilak et al. 2002; Chao et al. 2006). We have shown distinctly different chondrocyte deformations between femoral condyle and patellar samples (Table 1). More specifically, transverse (width, depth) strains are much higher in patellar cells compared to femoral condyle cells under equivalent compressive tissue loads (Madden et al. 2013). This could lead to differences in membrane expansion and unfolding events, both of which may be associated with calcium signaling via SACs that are activated primarily by membrane tension (Sachs
2010). Differences in cytoskeletal structure, which varies with depth and between load-bearing and non-load-bearing regions of joints (Eggli et al. 1988; Durrant et al. 1999), may also contribute to the regional differences in calcium signaling. Since integrin signaling is closely associated with the cell cytoskeleton, cytoskeletal differences could account for some of the differences in calcium signaling observed here (Loeser 2002; Millward-Sadler and Salter 2004). Patellar cartilage is also more permeable than femoral condyle cartilage (Froimson et al. 1997). This may result in differences in the fluid-velocity profile during the transient loading phase, which influences chondrocyte calcium signaling (Degala et al. 2012). The pericellular matrix (PCM) of cells affects the fluid flow around chondrocytes, thus differences in PCM structure and mechanical properties might also contribute to any differences between joint regions both in terms of deformation and calcium signaling (Guilak et al. 2006; Madden et al. 2013). Differences in chondrocyte cytoskeletal structure and PCM characteristics between joint regions should be investigated in future studies.

Calcium signal characteristics were moderately affected by the magnitude of compressive loading. Signal amplitude remained relatively consistent across all tissue loads for both joint regions, with the patellar cells exhibiting significantly shorter signaling events for the 10-30\% tissue strain conditions (Fig. 6). The lack of a significant difference at $40 \%$ tissue strain, despite marked differences in local mechanics, may again be a reflection of diminished mechanosensitivity (Donahue et al. 2003; Han et al. 2012). Calcium signal amplitude is a reflection of changes in intracellular $\mathrm{Ca}^{2+}$ and can provide clues as to the function of the calcium signal. For example, $\mathrm{IP}_{3}$ pathway opening is enhanced by modest increases in intracellular $\mathrm{Ca}^{2+}$ and inhibited by relatively large increases in $\mathrm{Ca}^{2+}$ (Berridge 1993; Bootman et al. 2001). The consistently higher signal amplitude in femoral condyle cells might indicate greater access to intracellular calcium stores. The decrease in signal amplitude at $40 \%$ nominal tissue strain was observed for both joint regions and may reflect a depletion of these intracellular calcium stores.

The duration of calcium signals remained consistent across all applied loads for femoral condyle cells. For patellar chondrocytes, there was an increase in signal duration with increasing load (Fig. 6). It has been shown that calcium signal duration differentially regulates specific genes in neuronallike CA77 cells (Durham and Russo 2000). However the role of signal duration in chondrocytes remains unclear. Duration is associated with the function of a calcium signal in terms of the distance to effector systems; longer signals can reach effector systems further away from the signal origin (Berridge et al. 2003). It is unlikely that the temporal differences observed here (5-10s) would significantly alter the effector systems reached as some calcium signals can be 
on the order of $10^{-6} \mu \mathrm{s}$ in other cell types (Berridge et al. 2003). Instead, the temporal differences between femoral condyle and patellar chondrocyte calcium signaling events may reflect signal tuning as a result of differing functional requirements and loading patterns of the respective cartilages, where structural differences have likely evolved and influence local cartilage properties (Little and Ghosh 1997; Herzog et al. 1998; Jurvelin et al. 2000; Treppo et al. 2000). Indeed, aggrecan synthesis rates differ between joint regions (Little and Ghosh 1997) and are associated with intracellular calcium (Fitzgerald et al. 2004). At $40 \%$ nominal tissue strain, the local ECM strain in patellae (44\%) is much greater than in femoral condyles (34\%, Table 1) (Madden et al. 2013). The significant difference in signal duration observed at $40 \%$ tissue strain may be a reflection of this large disparity in the local mechanical environments of the chondrocytes.

Compressive mechanical loading of intact articular cartilage resulted in calcium signals in the chondrocytes. The percentage of cells responding with calcium signaling tended to increase with increasing load magnitude $(p=0.133)$. Patellar samples appeared to exhibit more calcium signaling cells at each tissue load compared to femoral condyles, although this result was not significant likely due to the small number of patellae tested. The response times for calcium signaling agreed well with previous in situ studies, and they were faster than times reported for cell-gel constructs. This study provides new insight into calcium signaling in the intact cartilage attached to its native bone and further emphasizes the importance of studying chondrocytes in their natural environment. Future work using this in situ approach should investigate the anabolic or catabolic nature of the cellular response as it relates to calcium signaling and loading magnitude. Further studies will investigate the effect of loading magnitude on the calcium signaling behavior of chondrocytes from osteoarthritic cartilage, which undergo greater deformation than those in healthy tissue (Han et al. 2010). Currently, $\mathrm{OA}$ is a prevalent and irreversible joint disease with limited clinical treatment options. These results present an important step toward understanding the mechanisms underlying possible $\mathrm{Ca}^{2+}$-dependent signaling pathways that may be involved in cartilage homeostasis and the onset and progression of OA.

Acknowledgments Funded by the AI-HS Team Grant on Osteoarthritis, NSERC CREATE, CIHR, the Killam Memorial Chair and the Canada Research Chair for Molecular and Cellular Biomechanics. The authors would like to thank Azim Jinha and Andrzej Stano for technical support, and Dr. Tak-Shing Fung for assistance with the statistical analysis.

Open Access This article is distributed under the terms of the Creative Commons Attribution License which permits any use, distribution, and reproduction in any medium, provided the original author(s) and the source are credited.

\section{References}

Abusara Z, Seerattan R, Leumann A, Thompson R, Herzog W (2011) A novel method for determining articular cartilage chondrocyte mechanics in vivo. J Biomech 44(5):930-934

Amin A, Huntley J, Bush P, Simpson A, Hall A (2009) Chondrocyte death in mechanically injured articular cartilage-the influence of extracellular calcium. J Orthop Res 27(6):778-784

Berridge M (1993) Inositol trisphosphate and calcium signalling. Nature 361(6410):315-325

Berridge M, Bootman M, Roderick H (2003) Calcium signalling: dynamics, homeostasis and remodelling. Nat Rev Mol Cell Biol 4(7):517-529

Bootman M, Collins T, Peppiatt C, Prothero L, MacKenzie L, Smet PD, Travers M, Tovey S, Seo J, Berridge M, Ciccolini F, Lipp P (2001) Calcium signalling-an overview. Seminars Cell Develop Biol 12(1):3-10

Buschmann M, Kim Y, Wong M, Frank E, Hunziker E, Grodzinsky A (1999) Stimulation of aggrecan synthesis in cartilage explants by cyclic loading is localized to regions of high interstitial fluid flow. Arch Biochem Biophys 366(1):1-7

Chao P, West A, Hung C (2006) Chondrocyte intracellular calcium, cytoskeletal organization, and gene expression responses to dynamic osmotic loading. Am J Physiol Cell Physiol 291(4):C718-C725

Clark A, Votta B, Kumar S, Liedtke W, Guilak F (2010) Chondroprotective role of the osmotically sensitive ion channel transient receptor potential vanilloid 4: age- and sex-dependent progression of osteoarthritis in Trpv4-deficient mice. Arthr Rheum 62(10): 2973-2983

Clark C, Iannotti J, Misra S, Richards C (1994) Effects of thapsigargin, an intracellular calcium-mobilizing agent, on synthesis and secretion of cartilage collagen and proteoglycan. J Orthop Res 12(5):601-611

Degala S, Zipfel W, Bonassar L (2011) Chondrocyte calcium signaling in response to fluid flow is regulated by matrix adhesion in 3-D alginate scaffolds. Arch Biochem Biophys 505(1):112-117

Degala S, Williams R, Zipfel W, Bonassar L (2012) Calcium signaling in response to fluid flow by chondrocytes in $3 \mathrm{D}$ alginate culture. $\mathrm{J}$ Orthop Res 30(5):793-799

Donahue S, Donahue H, Jacobs C (2003) Osteoblastic cells have refractory periods for fluid-flow-induced intracellular calcium oscillations for short bouts of flow and display multiple low-magnitude oscillations during long-term flow. J Biomech 36(1):35-43

Durham P, Russo A (2000) Differential regulation of mitogen-activated protein kinase-responsive genes by the duration of a calcium signal. Mol Endocrinol 14(10):1570-1582

Durrant L, Archer C, Benjamin M, Ralphs J (1999) Organisation of the chondrocyte cytoskeleton and its response to changing mechanical conditions in organ culture. J Anat 194(Pt. 3):343-353

Eggli P, Hunziker E, Schenk R (1988) Quantitation of structural features characterizing weight- and less-weight-bearing regions in articular cartilage: A stereological analysis of medial femoral condyles in young adult rabbits. Anatom Rec 222(3):217-227

Erickson G, Northrup D, Guilak F (2003) Hypo-osmotic stress induces calcium-dependent actin reorganization in articular chondrocytes. Osteoarth Cartil 11(3):187-197

Fitzgerald J, Jin M, Dean D, Wood D, Zheng M, Grodzinsky A (2004) Mechanical compression of cartilage explants induces multiple timedependent gene expression patterns and involves intracellular calcium and cyclic AMP. J Biol Chem 279(19):19502-19511

Froimson M, Ratcliffe A, Gardner T, Mow V (1997) Differences in patellofemoral joint cartilage material properites and their significance to the etiology of cartilage surface fibrillation. Osteoarth Cartil 5(6):377-386

Guilak F, Zell R, Erickson G, Grande D, Rubin C, McLeod K, Donahue $\mathrm{H}$ (1999) Mechanically induced calcium waves in articular chon- 
drocytes are inhibited by gadolinium and amiloride. J Orthop Res 17(3):421-429

Guilak F, Erickson G, Ting-Beall H (2002) The effects of osmotic stress on the viscoelastic and physical properties of articular chondrocytes. Biophys J 82(2):720-727

Guilak F, Alexopoulos L, Upton M, Youn I, Choi J, Cao L, Setton L, Haider M (2006) The pericellular matrix as a transducer of biomechanical and biochemical signals of articular cartilage. Ann N Y Acad Sci 1068:498-512

Han SK, Colarusso P, Herzog W (2009) Confocal microscopy indentation system for studying in situ chondrocyte mechanics. Med Eng Phys 31(8):1038-1042

Han SK, Seerattan R, Herzog W (2010) Mechanical loading of in situ chondrocytes in lapine retropatellar cartilage after anterior cruciate ligament transection. J Roy Soc Interf 7(47):895-903

Han SK, Wouters W, Clark A, Herzog W (2012) Mechanically induced calcium signaling in chondrocytes in situ. J Orthop Res 30(3):475481

Herberhold C, Faber S, Stammberger T, Steinlechner M, Putz R, Englmeier K, Reiser M, Eckstein F (1999) In situ measurement of articular cartilage deformation in intact femoropatellar joints under static loading. J Biomech 32(12):1287-1295

Herzog W, Diet S, Suter E, Mayzus P, Leonard T, Muller C, Wu J, Epstein M (1998) Material and functional properties of articular cartilage and patellofemoral contact mechanics in an experimental model of osteoarthritis. J Biomech 31(12):1137-1145

Huser C, Davies M (2007) Calcium signaling leads to mitochondrial depolarization in impact-induced chondrocyte death in equine articular cartilage explants. Arthr Rheum 56(7):2322-2334

Jurvelin J, Arokoski J, Hunziker E, Helminen H (2000) Topographical variation of the elastic properties of articular cartilage in the canine knee. J Biomech 33(6):669-675

Kono T, Nishikori T, Kataoka H, Uchio Y, Ochi M, Enomoto K (2006) Spontaneous oscillation and mechanically induced calcium waves in chondrocytes. Cell Biochem Func 24(2):103-111

Little C, Ghosh P (1997) Variation in proteoglycan metabolism by articular chondrocytes in different joint regions is determined by postnatal mechanical loading. Osteoarthr Cartil 5(1):49-62

Loeser R (2002) Integrins and cell signaling in chondrocytes. Biorheology 39(1-2):119-124

Lu X, Huo B, Chiang V, Guo X (2012) Osteocytic network is more responsive in calcium signaling than osteoblastic network under fluid flow. J Bone Miner Res 27(3):563-574

Madden R, Han SK, Herzog W (2013) Chondrocyte deformation under extreme tissue strain in two regions of the rabbit knee joint. J Biomech 46(3):554-560

Millward-Sadler S, Salter D (2004) Integrin-dependent signal cascades in chondrocyte mechanotransduction. Ann Biomed Eng 32(3):435446

Millward-Sadler S, Wright M, Lee HS, Nishida K, Caldwell H, Nuki G, Salter D (1999) Integrin-regulated secretion of Interleukin 4: a novel pathway of mechanotransduction in human articular chondrocytes. J Cell Biol 145(1):183-189

Mizuno S (2005) A novel method for assessing effects of hydrostatic fluid pressure on intracellular calcium: a study with bovine articular chondrocytes. Am J Physiol Cell Physiol 288(2):C329-C337
Mobasheri A, Carter S, Martin-Vasallo P, Shakibaei M (2002) Integrins and stretch activated ion channels; putative components of functional cell surface mechanoreceptors in articular chondrocytes. Cell Biol Int 26(1):1-18

Pingguan-Murphy B, Lee D, Bader D, Knight M (2005) Activation of chondrocytes calcium signalling by dynamic compression is independent of number of cycles. Arch Biochem Biophy 444(1):45-51

Pingguan-Murphy B, El-Azzeh M, Bader D, Knight M (2006) Cyclic compression of chondrocytes modulates a purinergic calcium signalling pathway in a strain rate- and frequency-dependent manner. J Cell Physiol 209(2):389-397

Ralevic V, Burnstock G (1998) Receptors for purines and pyrimidines. Pharmacol Rev 50(3):413-492

Roberts S, Knight M, Lee D, Bader D (2001) Mechanical compression influences intracellular $\mathrm{Ca} 2+$ signaling in chondrocytes seeded in agarose constructs. J Appl Physiol 90(4):1385-1391

Sachs F (2010) Stretch-activated ion channels: what are they? Physiology 25(1):50-56

Sah R, Kim YJ, Doong J, Grodzinsky A, Plaas A, Sandy J (1989) Biosynthetic response of cartilage explants to dynamic compression. J Orthop Res 7(5):619-636

Shen J, Luscinskas F, Connolly A, Jr CD, Jr MG (1992) Fluid shear stress modulates cytosolic free calcium in vascular endothelial cells. American Journal of Physiology-Cell Physiology 262(2 (Part 1)):C384-C390

Treppo S, Koepp H, Quan E, Cole A, Kuettner K, Grodzinsky A (2000) Comparison of biomechanical and biochemical properties of articular cartilage from human knee and ankle pairs. J Orthop Res 18(5):739-748

Valhmu W, Raia F (2002), myo-Inositol 1,4,5-trisphosphate and $\mathrm{Ca} 2+/$ calmodulin-dependent factors mediate transduction of compression-induced signals in bovine articular chondrocytes. Biochemical Journal 361(Part 3):689-696

Wann A, Zuo N, Haycraft C, Jensen C, Poole C, McGlashan S, Knight M (2012) Primary cilia mediate mechanotransduction through control of ATP-induced $\mathrm{Ca} 2+$ signaling in compressed chondrocytes. FASEB J 26(4):1663-1671

Wieland H, Michaelis M, Kirschbaum B, Rudolphi K (2005) Osteoarthritis-an untreatable disease? Nat Rev Drug Discov 4(4):331-344

Wright M, Jobanputra P, Bavington C, Salter D, Nuki G (1996) Effects of intermittent pressure-induced strain on the electrophysiology of cultured human chondrocytes: evidence for the presence of stretchactivated membrane ion channels. Clin Sci (London) 90(1):61-71

Wright M, Nishida K, Bavington C, Godolphin J, Dunne E, Walmsley S, Jobanputra P, Nuki G, Salter D (1997) Hyperpolarisation of cultured human chondrocytes following cyclical pressure-induced strain: evidence of a role for $\alpha 5 \beta 1$ integrin as a chondrocyte mechanoreceptor. J Orthop Res 15(5):742-747

Yellowley C, Jacobs C, Li Z, Zhou Z, Donahue H (1997) Effects of fluid flow on intracellular calcium in bovine articular chondrocytes. Am J Physiol Cell Physiol 273(1):C30-C36 